\title{
Use of penile prostheses to maintain external condom catheter drainage in spinal cord injury patients
}

\author{
I Perkash MD, J N Kabalin MD, S Lennon RN, V Wolfe RN \\ Department of Urology, Stanford University and Spinal Cord Injury Service, Department \\ of Veterans Affairs Medical Centers, Palo Alto, California, USA.
}

This is a retrospective analysis of 79 spinal cord injury patients who have had penile implants from one to 14 years. The primary indication for implants was the loss of condom catheter with a small retractile penis. Mean period after injury to when the implants were placed was 8.24 years (range 1-21 years). Mean total length of time the implants have been followed was 7.08 years (1-14 years). Sixty patients responded to our detailed questionnaire and they have been subjected to further analysis: prior to the implant 46 patients $(77 \%)$ frequently lost their condoms. Fourteen of the patients $(23 \%)$ had indwelling catheters, and $3(5 \%)$ had a suprapubic cystostomy since they could not retain an external condom for urinary drainage because of retraction of a small penis. Post implant, $81 \%$ of patients had no accidents involving condom loss, while $19 \%$ still lost condoms. All indwelling catheters could be removed except for one patient who continued with a suprapubic catheter following transurethral sphincterotomy (TURS) and a penile implant. Sixty-eight percent used the implant for sex and felt their wives were satisfied. Patient satisfaction survey showed a markedly increased self esteem, increased mobility without fear of condom loss, and an improved sex life. Overall, the long term prosthesis failure rate was $8 \%$. The specific infection complication rate was less than $2 \%$. The Flexirod semirigid, hinged prosthesis proved ideal in meeting the requirements for these patients.

Key words: penile prosthesis; neuropathic bladder; spinal cord injury.

\section{Introduction}

Semirigid penile implants have been found to be useful in the rehabilitation of spinal cord injury patients who have a small retractile phallus. ${ }^{1}$ In general, their spouses have been satisfied with the results of penile prosthesis implantation. ${ }^{2,3}$ Penile implants provide a stable penile shaft to hold a condom for external urinary drainage, improve sexual relationships with partners, and help to reduce the condition of skin maceration. ${ }^{4}$ However, the incidence of infection and implant failure has been much higher in spinal cord injury patients when compared with non paralyzed patients - up to $33 \%$ versus $8.3 \%$ respectively. ${ }^{2-8}$ We present our experience with 79 spinal cord injury patients who had implants in the past 14 years with an overall failure rate of $8 \%$.

\section{Methods and materials}

Seventy-nine spinal cord injury patients who had had implants in the past 14 years were reviewed. The mean age was 41.9 years (range 20 to 74 years). Primary indication for implants was inability to hold external condom drainage over a small retractile phallus. There were $38(48 \%)$ tetraplegics, $37(47 \%)$ paraplegics and $4(5 \%)$ patients who had cauda equina lesions. Sixty-one $(77 \%)$ were neurologically complete (Frankel A), and 18 (23\%) were incomplete lesions (Frankel C and D). Prior to implantation, urodynamic evaluation and transurethral resection of the sphincter (TURS) and/or transurethral resection of the prostate (TURP) were done to optimize bladder drainage. At least a week prior to the implantation, daily urinary cultures were 
obtained and patients with positive cultures were treated with appropriate antibiotics to sterilize the urine. Patients were operated on only when at least 2 days prior to surgery urine cultures did not show any colony forming organisms. Initially, 5 patients were operated through a perineal incision, with wound dehiscence occurring in 3 of these patients. The remaining 74 patients had an infrapubic midline approach, not extending onto the base of the penis so that the indwelling catheter could be removed within 24 hours post surgery and external condom drainage could be applied. Thirty-nine patients $(49 \%)$ had Small Carrion prostheses, ${ }^{9}$ $36(45 \%)$ patients had Flexi-rod hinge-type prostheses, ${ }^{10}$ and 4 had other types of implants.

\section{Results}

Seventy-nine patients had had penile implants for one to 14 years, placed within a mean period of 8.24 years after their spinal cord injury. The mean total length of time the implants have been in place is 7.08 years. All patients were followed for at least every 6 months during the first year after surgery, and yearly thereafter. Six patients (3\%) died due to unrelated causes, one to 10 years post implant.

Twenty-nine patients (49\%) had Small Carrion prostheses, and $36(45 \%)$ had Flexirod hinge-type prostheses and 4 had other types implants. The placement of a penile prosthesis generally provided adequate length of shaft and penile rigidity both to hold a condom and for sexual penetration (Figs 1a, 1b, 2). Three patients who were obese had severe retraction of the penis and were reimplanted after mobilizing the corpora cavernosa, releasing the suspensory ligament of the penis, and placing buttress sutures behind the corpora to prevent retraction. We have recently reported this technique for penile advancement and lengthening in patients with retracted penis who still retracted and where condoms could not be retained despite the placement of a penile prosthesis. ${ }^{11}$

The complications immediately following surgery included one patient with an infection with loss of the implant within 2

\section{$\mathbf{a}$}
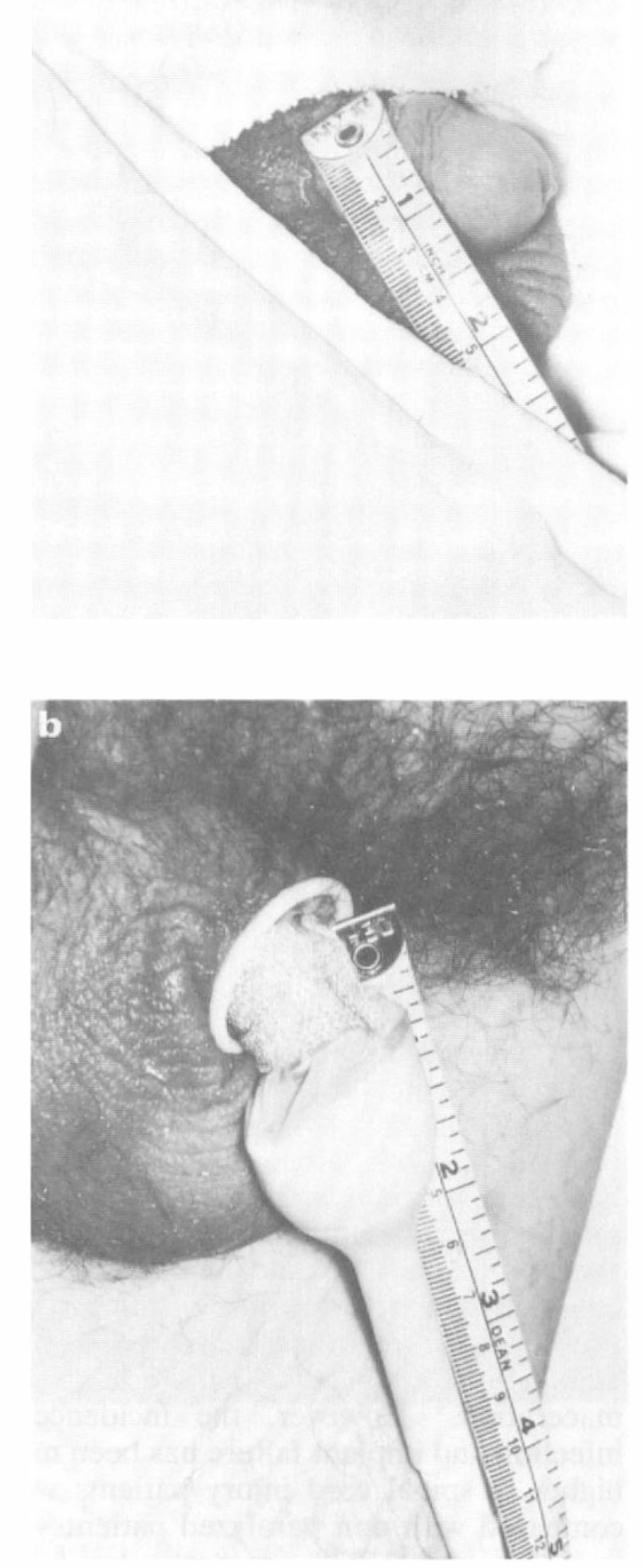

Figure 1 Preoperative size of the phallus (a) without and (b) with external condom urinary drainage. 


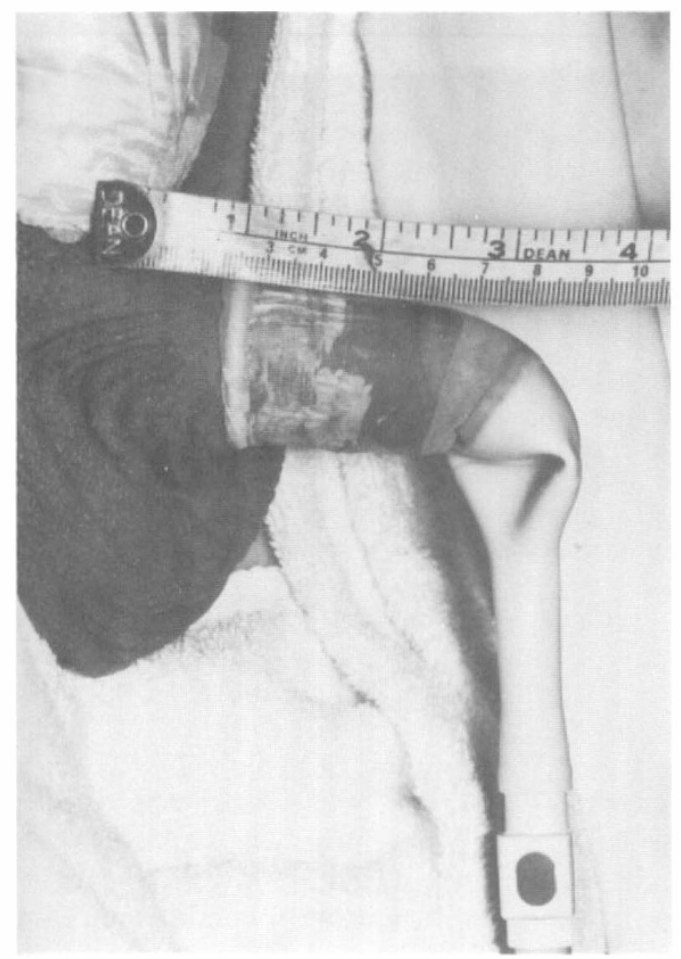

Figure 2 Postoperative size of the phallus following penile implant with external condom drainage. There is significant increase in the length of the penis and easy application of condom drainage.

months. This was the only patient who had a positive urine culture 2 days prior to implant and was treated with aminoglycosides and cephalosporin prior to surgery but the urine was not sterilized. His penile implants were removed about 2 months after implantation. Five other patients lost implants (see Table I). In one patient the implant was removed because of causalgia (T12 lesion). Two implants were lost after the patients had indwelling Foley catheters for several weeks during the treatment of pressure sores in other hospitals. One patient was further complicated by prolonged prone positioning, and in another the implant was lost following circumcision and inadvertent placement of an external condom by the patient for urinary drainage only 24 hours after the circumcision, leading to gangrene of the glans penis. In another patient, return of severe spasticity, despite dorsal rhizotomy, resulted in extrusion of one implant about 6 months after placement. The overall failure rate was $8 \%$ with an infection complication rate of less than $2 \%$.

The Quality of Life Questionnaire survey was responded to by 60 of the 73 living patients $(80 \%)$, and they have been further analyzed: 29 (48\%) were tetraplegics, 28 $(47 \%)$ were paraplegics and $3(5 \%)$ had cauda equina lesions. Forty-eight patients $(80 \%)$ were complete (Frankel A) and 12 $(20 \%)$ were incomplete lesions (Frankel C and D). Fifty patients (83\%) had transurethral resection of sphincter and/or transurethral resection of the prostate prior to the implants. Six of them $(10 \%)$ had repeat transurethral surgery after the implants without any damage to the penile implants.

Results of the satisfaction survey showed that prior to placement of penile implants, 46 patients $(77 \%)$ lost condoms and had accidents with urine leakage more than twice a week. There were $11(18 \%)$ who had indwelling catheters since they could not retain an external collecting device at all, and $3(5 \%)$ had suprapubic cystostomies. After implantation, $81 \%$ had no problems, and $19 \%$ lost condoms once or twice a week. In all of the patients with an indwelling catheter the penile prosthesis was successful in that the catheter was no longer necessary. Two of 3 patients with suprapubic catheters were also successfully managed. Sixty-eight percent of the patients were able to have sexual intercourse and their wives were satisfied. All patients felt that it was easier to keep themselves clean and dry and it took less time to apply the external condom drainage after penile implants were in place. Their mobility was increased without the fear of urinary leakage. Seventy-nine percent of our patients felt that the penile shaft after the implant was excellent for maintaining condom drainage.

\section{Discussion}

The value of penile implants in patients with a neuropathic bladder has already been reported. They permit external condom 
Table 1 Complications leading to loss of implants in 6 patients

\begin{tabular}{lccl}
\hline Level of injury & $\begin{array}{c}\text { Month/year } \\
\text { implanted }\end{array}$ & $\begin{array}{c}\text { Month/year } \\
\text { lost }\end{array}$ & Possible causes \\
\hline C5-6 & $5 / 89$ & $7 / 89$ & Perioperative infection \\
T-12 & $7 / 83$ & $7 / 85$ & $\begin{array}{l}\text { Causalgia } \\
\text { T4-5 }\end{array}$ \\
& $6 / 77$ & $6 / 87$ & $\begin{array}{l}\text { Nursed prone following plastic surgery with } \\
\text { indwelling urethral catheter }\end{array}$ \\
T-4 & $3 / 87$ & $10 / 88$ & $\begin{array}{l}\text { Indwelling urethral catheter } \\
\text { L-1 }\end{array}$ \\
C5-6 & $2 / 83$ & $8 / 83$ & $\begin{array}{l}\text { Post circumcision } \\
\text { Severe spasticity. Lost implant } 6 \text { months } \\
\end{array}$ \\
& & & after surgery \\
\hline
\end{tabular}

drainage and so maintain continence, prevent skin maceration in a small retractile penis needing glue and other sticking material to retain condoms, and allow vaginal penetration for sexual satisfaction. They have also been reported to be useful to facilitate intermittent self catheterization by lengthening and straightening the penis. ${ }^{12}$

The use of prostheses in the management of impotence is now widely accepted for diabetic and other patients. Several series have reported both technical and mechanical complications ${ }^{9}$ and others have specifically reported infectious complication rates both in spinal cord and non spinal cord injury patients. ${ }^{8,13,14}$ However, overall the reported complication rates leading to extrusion of implants are significantly higher in neurologically impaired patients, ranging from $16.5 \%$ in one series ${ }^{5}$ to $33 \%$ implant failure in another series dealing with spinal cord injury patients. ${ }^{7}$ As a result, great caution has been advised in selecting such patients for penile implants. Loss of implants due to pressure necrosis of the skin and corporeal bodies has been reported when patients were placed prone for long periods of time for the treatment of pressure ulcers, ${ }^{7}$ as happened in one of our patients. Bilateral ischiectomies, which are rarely performed nowadays, have also been associated with extrusion of implants when patients virtually sat upon the corpora on their wheelchairs. ${ }^{7}$ Transurethral procedures, such as sphincterotomy and cystolithotripsy, have also resulted in extrusion of implants due to trauma to the urethra. ${ }^{5}$
The present series of spinal cord injury patients with neurogenic bladder dysfunction, when considered for penile implants, were evaluated with urodynamics and treated prior to implantation with TURS and/ or TURP so that the need for future transurethral procedures was reduced. We made a point of removing indwelling catheters within 24 hours after penile implant surgery in all of our patients to minimize urethral inflammation and compression, and hopefully thus the incidence of erosion. We also purposely placed smaller diameter implants in our patients, usually $9 \mathrm{~mm}$ diameter. In $10 \%$ of our patients subsequent TURS or TURP was required several years after the initial implant surgery. The smaller diameter implants allowed subsequent introduction of a smaller resectoscope sheath into the urethra with minimal trauma. Thus, we did not experience any complications related to the prostheses in this subgroup of patients requiring late transurethral surgery.

Our earlier patients with penile implants inserted through a perineal incision were complicated by disruption of the incision when patients transferred from bed to wheelchair and vice versa. The surgical approach of choice was therefore changed. We now use an infrapubic incision at the base of the penis. The incision does not extend onto the upper surface of the shaft of the penis. Thus, it is easy to begin external condom drainage on the day following surgery. At surgery penile implants are properly sized.

Since implants were used to promote 
constant adequate shaft for condom drainage, semirigid implants were a preference in this series. In an attempt to hold condom drainage onto a retractile small penis, the application of extra glue and/or tightly placed tapes results in laceration of the skin. It has been reported by Van Arsdalen et al ${ }^{4}$ that in 14 of their patients with frequent skin lacerations following penile implants, only 3 had penile skin problems. Frequently the loss of condoms leads to wetness and the penile skin macerates. Semirigid implants provide length all the time with much less concern over losing the condom. Patients therefore tend to apply less glue or non tight tapes to hold the condom. The Finney hinge type $^{10}$ of prosthesis has been found optimal and was less prominent than the Carrion Small prosthesis. ${ }^{9}$ Hydraulic cylinder and inflatable implant devices are also not considered very suitable in tetraplegics who lack hand function, since they require finger dexterity to activate or deactivate the device. Mechanical failures are more likely to happen with inflatable implants than with semirigid implants. ${ }^{14}$

Key factors for our lower failure rate seem to be adequate bladder drainage, and sterilization of urinary tract infection prior to implant surgery. Early removal of the indwelling catheter following implantation has helped in the prevention of local urethral infection and implant erosion.

\section{Conclusion}

The beneficial results of semirigid penile implants in the urological and sexual rehabilitation of spinal cord injury patients are presented. Infectious complications leading to loss of such implants can be reduced by careful monitoring, and the eradication of urinary tract infection and contamination beginning at least one week prior to the implant surgery. Using this protocol, loss of implant due to infection occurred in only one patient out of 79 in this series (representing less than a $2 \%$ overall infectious complication rate). Urinary drainage was optimized with transurethral resection of the sphincter and/or transurethral resection of the prostate prior to implantation. Assuring adequate bladder drainage prior to implantation both minimizes the subsequent incidence of bacteriuria and decreases the need for future transurethral manipulation and instrumentation; the latter is not only limited by the presence of the prosthesis but also may allow trauma to the urethra and/or prosthesis as well as bacteremic seeding of the implant. While early loss of prostheses seems to be related primarily to perioperative infection, later extrusions are more commonly related to local pressure necrosis in spinal cord injury patients. Good medical and nursing management of these patients after penile implantation can minimize loss of the prosthesis. In particular, care should be taken to avoid undue external compression of the penis (especially as might occur with the prone positioning of insensate patients for care of decubiti), patients should receive antibiotic prophylaxis for all subsequent non clean surgical procedures (including dental/oral, genitourinary, and gastrointestinal), and urethral catheterization should be limited. When an indwelling urethral catheter is required, selection of a smaller diameter catheter (16 french maximum, but preferably 12 or 14 french), will limit urethral compression and trauma and thus the chance of erosion of the prosthesis. Even with a smaller catheter, the period of urethral catherization should be limited to 24 to 48 hours whenever possible. For patients requiring an indwelling urethral catheter in excess of 72 hours, placement of a temporary percutaneous suprapubic catheter is recommended.

\section{References}

1 Melman A, Hammond B (1978) Placement of the Small-Carrion penile prosthesis to enable maintenance of an exdwelling condom catheter. Sex Disabil 1: 292.

2 Light JK, Scott FB (1981) Management of neurogenic impotence with inflatable penile prosthesis. Urology 17: 341 .

3 Golgi H (1979) Experience with penile prosthesis in spinal cord injury patients. J Urol 121: 288. 
4 Van Arsdalen KN, Klein FA, Hackler RH, Brady SM (1981) Penile implants in spinal cord injury patients for maintaining external appliances. J Urol 126: 331.

5 Rossier AB, Fam BA (1984) Indication and results of semirigid penile prostheses in spinal cord injury patients: long-term followup. J Urol 131: 59-62.

6 Iwatsubo E, Tanaka M, Takahashi K, Akatsu T (1986) Non-inflatable penile prosthesis for the management of urinary incontinence and sexual disability of patients with spinal cord injury. Paraplegia 24: 307-310.

7 Collins K, Hackler RH (1988) Complications of penile prostheses in the spinal cord injury population. $J$ Urol 140: 984-985.

8 Thomalla JV, Thompson ST, Rowland RG, Mulcahy JJ (1987) Infectious complications of penile prosthetic implants. J Urol 138: 65-67.

9 Chaikin L, Carrion H, Politano V (1981) Complications of the Small-Carrion penile prosthesis: Long-term followup. J Urol 126: 44-45.

10 Finney RP, Sharpe JR, Sadlowski RW (1980) Finney hinged penile implant: experience with 100 cases. $J$ Urol 124: 205-207.

11 Kabalin JN, Rosen J, Perkash I (1990) Penile advancement and lengthening in spinal cord injury patients with retracted phallus who have failed penile prosthesis placement alone. J Urol 144: 316-318.

12 Smith AD, Sazama R, Lange PH (1980) Penile prosthesis: adjunct to treatment in patients with neurogenic bladder. J Urol 124: 363-64.

13 Kabalin JN, Kessler R (1988) Infectious complications of penile prosthesis surgery. J Urol 139: 953.

14 Kabalin JN, Kessler R (1988) Five year followup of the Scott inflatable penile prosthesis and comparison with semirigid penile prosthesis. J Urol 140: 1428. 